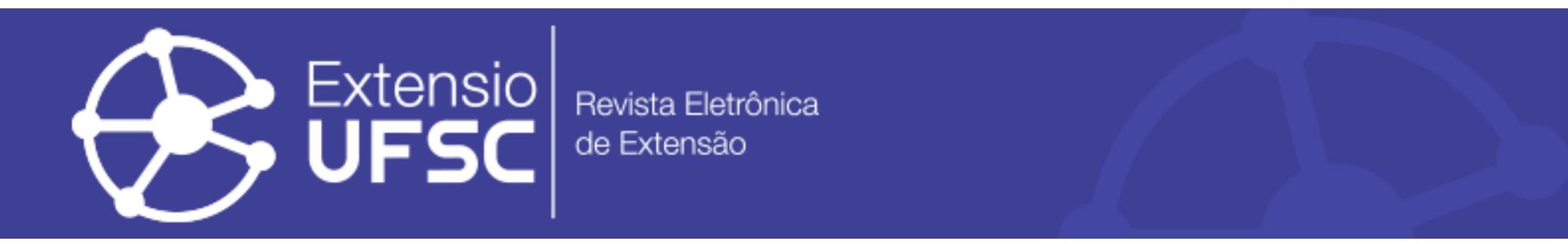

\title{
TECNOLOGIAS LEVES PARA O CUIDADO DE ENFERMAGEM NA ATENÇÃO PSICOSSOCIAL: CONTRIBUIÇÕES À SUPERAÇÃO DE ESTIGMAS SOBRE A DOENÇA MENTAL
}

Valquiria Farias Bezerra Barbosa
Instituto Federal de Pernambuco
valquiriaenfermeira@yahoo.com.br

Cleomarcos Gomes Lima

Instituto Federal de Pernambuco

cleo_marcos@hotmail.com
July Polyana de Souza Simões Instituto Federal de Pernambuco julypolyana_@hotmail.com

Robervam de Moura Pedroza Instituto Federal de Pernambuco robervam@pesqueira.ifpe.edu.br

Marcelle Guimarães de Mello Instituto Federal de Pernambuco marcellegmello@gmail.com

\section{Resumo}

OBJETIVO: Desenvolver intervenções com vistas à superação de concepções estigmatizantes sobre o processo saúdeadoecimento mental, vivenciando a atuação do enfermeiro no âmbito da atenção psicossocial. METODOLOGIA: Tratase de relato de experiência descritivo, de abordagem qualitativa, realizado no município de Pesqueira, PE no período de fevereiro a dezembro de 2015. RESULTADOS: Desenvolvemos intervenções psicossociais contribuindo para discussões sobre o estigma com os profissionais de saúde e usuários de uma unidade básica de saúde. Promovemos a conscientização sobre o uso indiscriminado de medicamentos psicotrópicos e a abertura para novos modelos de atenção à saúde, como as práticas integrativas e complementares. CONCLUSÕES: A estigmatização da loucura faz com que seja negada à pessoa em sofrimento psíquico sua cidadania. A ressocialização ainda é difícil pois a doença mental é interpretada como transgressão de normas sociais, o que demanda das equipes multiprofissionais de saúde intervenções no território e com a comunidade.

Palavras-chave: Cuidados de Enfermagem. Atenção Primária de Saúde. Saúde Mental. Desinstitucionalização.

\section{LIGHT TECHNOLOGIES FOR NURSING CARE IN PSYCHOSOCIAL ATTENTION: CONTRIBUTIONS TO OVERCOMING STIGMAS ON MENTAL DISEASE}

Abstract

AIM: To develop intersectoral interventions fot overcoming stigmatizing conceptions of mental health-disease process, experiencing the role of the nurse in the context of psychosocial care. METHODOLOGY: This is a descriptive experience report of qualitative approach, implemented in the city of Pesqueira, PE, Brazil, from February to December 2015. RESULTS: We developed psychosocial interventions contributing to discussions on stigma with health professionals and users of a basic health unit. We promoted awareness of the indiscriminate use of psychotropic drugs and openness to new health care models, such as complementary and integrative practices. CONCLUSIONS: The stigma of madness makes it denied to the person in mental suffering their citizenship. The rehabilitation is still difficult because mental illness is interpreted as transgressing social norms, which demand of multidisciplinary health team's interventions in the territory and with the community.

Keywords: Nursing Care. Primary Health Care. Mental Health. Deinstitutionalization.

\section{TECNOLOGÍAS LEVES PARA EL CUIDADO DE ENFERMERÍA EN LA ATENCIÓN PSICOSOCIAL: CONTRIBUCIONES A LA SUPERSIÓN DE ESTIGMAS SOBRE LA ENFERMEDAD MENTAL}

Resumen

OBJETIVO: Desarrollar intervenciones dirigidas a la superación de las concepciones estigmatizantes del proceso saludenfermedad mental y experimentar el trabajo de enfermeria en atención psicosocial. METODOLOGÍA: Relato de experiencia descriptivo de enfoque cualitativo, llevado a cabo en el municipio de Pesqueira, PE, Brazil, desde febrero a diciembre de 2015. RESULTADOS: Desarrollamos intervenciones psicosociales que contribuyen a los debates sobre el estigma con profesionales de la salud y usuarios em una unidad básica de salud. Promovemos la conciencia del uso indiscriminado de drogas psicotrópicas y la apertura a nuevos modelos de atención de la salud, tales como prácticas complementarias e integradoras. CONCLUSIONES: El estigma de la locura hace que sea negada a la persona que sufre su ciudadanía. La rehabilitación sigue siendo difícil debido a las interpretaciones de la enfermedad mental como transgresión de normas sociales y los equipos multidisciplinarios de salud deveran desarollar intervenciones en el territorio y por la comunidad.

Palabras clave: Atención de Enfermería. Atención Primaria de Salud. Salud Mental. Desinstitucionalización. 
Tecnologias leves para o cuidado de enfermagem na atenção psicossocial: contribuições à superação de estigmas sobre a doença mental

\section{INTRODUÇÃO}

Os portadores de transtorno mental foram tratados, por muito tempo, como loucos, perigosos, libertinos e, portanto, alijados da sociedade e do próprio convívio com a família, tornando-se muitas vezes mendigos ou moradores dos hospícios (MARTINS et.al, 2013).

Essa visão sobre os portadores de transtornos mentais contribuiu para o desenvolvimento do estigma, que, ao inserí-lo na condição de diferente, interfere nas suas relações com a sociedade e no acesso aos ambientes sociais. O estigma ao portador de transtorno mental, ainda percebido nos dias de hoje, pode ser entendido como situação onde o indivíduo que está inabilitado para a aceitação social de forma integral (MARTINS et.al, 2013, p.328).

De acordo com Corrigan e Watson (2002), existem dois tipos de estigma: o estigma público e o auto estigma. O estigma público é a percepção de um grupo ou da sociedade de que um indivíduo é socialmente inaceitável, devido a características físicas ou pessoais muitas vezes associadas a reações negativas e, consequentemente, à desvalorização do indivíduo. Já o estigma internalizado ocorre à medida que o indivíduo em condição estigmatizante torna-se consciente dos estereótipos negativos sobre seu transtorno, concorda com eles e os aplica a si próprio (SOARES et. al, 2011).

Nesse sentido, a participação de pessoas estranhas àquelas que, hegemonicamente, habitam um espaço social, permite aos que se identificam como normais que categorizem os estranhos, conforme seus atributos e características, e, assim, estabelecem sua "identidade social" (MARTINS et.al, 2013).

O estigma pode ser expresso como uma condição genérica de preconceito arraigado e naturalizado na nossa cultura (SPADINI; SOUZA, 2006, p.125). Esse preconceito mantém-se relacionado, principalmente, aos conceitos de periculosidade e de infantilidade atribuídos à loucura e a uma redução do sujeito à doença. O estigma também atinge por extensão a família do doente, levando-a a um isolamento progressivo da vida social, tendo como consequência a sobrecarga do cuidador.

De fato, desde o texto da I Conferência Nacional de Saúde Mental (CNSM) percebemos expressões de preocupação quanto à superação dos estigmas impostos ao sujeito pelo fato de seu adoecimento ou de seu sofrimento psíquico. Essa preocupação implicou a adoção paulatina de outras formas menos estigmatizantes de se referir a esse sujeito, ao ponto de encontrarmos, na IV CNSM-I, uma recomendação explícita de que seja adotada a nomenclatura "pessoa em sofrimento psíquico", para designar os usuários dos serviços de saúde mental em todo o texto de seu relatório final (BARBOSA, 2014). 
Tecnologias leves para o cuidado de enfermagem na atenção psicossocial: contribuições à superação de estigmas sobre a doença mental

As mídias, em suas variadas manifestações, operam como lugar de reforço dos estigmas. No entanto, parece-nos que uma outra possibilidade se vislumbra: a de nelas encontrar a transposição (ruptura) de estigmas sociais. Os discursos midiáticos, em geral, e os discursos jornalísticos, em particular, encontram-se repletos de expressões cheias de preconceitos e que carecem de explicação (SOARES, 2009).

A estigmatização da loucura faz com que a pessoa doente tenha a sua cidadania negada, sofrendo preconceitos e segregação social. Hoje é sabido que a doença mental, de gênese multicausal, requer assistência adequada, com a finalidade de ressocialização do indivíduo em sofrimento psíquico e de apoio adequado para este e para a sua família. No entanto, a ressocialização ainda é difícil, pois a doença mental, em alguns casos, ainda é vista como transgressão de normas sociais (SPADINI; SOUZA, 2006).

Assim, é importante ressaltar a necessidade de esclarecimento da população sobre o processo saúde-adoecimento mental para que os preconceitos e estigmas diminuam. Para construir a inclusão social da pessoa com transtornos mentais na sociedade é preciso lidar com a herança cultural, que estabelece o conceito de que as pessoas com transtornos mentais devem ser temidas e excluídas, enfrentando preconceitos e criando novas possibilidades de se compreender o adoecimento mental (NUNES; TORRENTÉ, 2009).

O fato da pessoa com transtorno mental viver na comunidade não significa que esteja incluída e que faça parte dessa comunidade. Para o alcance da inclusão social, é preciso a reconstrução de uma vida significativa e satisfatória, na qual se tenha acesso às oportunidades relacionadas a diferentes atividades e papéis sociais que considerem importantes (BARBOSA, 2014).

Outra maneira de mudar as concepções herdadas culturalmente, é ter exemplos de pessoas com transtornos mentais que conseguiram se reinserir na sociedade e vivenciam outros projetos de vida envolvendo trabalho, estudo, cuidar da casa, filhos ou parentes. Assim é possível demonstrar para a sociedade uma nova representação da loucura a partir da experiência (SALLES; BARROS, 2013).

A integração na comunidade ainda continua um desafio para as pessoas com transtornos mentais, pois quando uma pessoa recebe o rótulo de ter transtorno mental severo, poderá ser inserida num processo de exclusão social em que, frequentemente, lhe são negadas oportunidades e direitos, em geral, garantidos para as outras pessoas da sociedade. Assim, um transtorno mental prolongado estará associado não apenas ao sofrimento, mas também à perda da liberdade, da autonomia e do acesso a recursos materiais e sociais (SALLES; BARROS, 2013). 
Tecnologias leves para o cuidado de enfermagem na atenção psicossocial: contribuições à superação de estigmas sobre a doença mental

Em pesquisa desenvolvida por Barbosa (2014, p.198) os usuários dos Centros de Atenção Psicossocial II (CAPS) Cultivando Sorrisos, localizado em Pesqueira, PE, relataram sobre o estigma e preconceito que sofrem quando, ao circularem pela cidade ou por seu bairro, muitas vezes em seu trajeto diário ao CAPS, são chamados de "psicopatas", "doidos", entre outros. Uma frase dita por um usuário retratou de forma bastante clara esse conflito relacionado à circulação do "doente mental" pelo território nesse contexto local: "É fácil ficar perto de quem é normal" (BARBOSA, 2014, p.198).

A circulação do "doente mental" não é, portanto, totalmente livre no território, mas negociada ou mesmo imposta. Há uma negociação velada entre família e "comunidade" sobre em quais espaços sociais essa pessoa "doente" será aceita. Dizendo de outro modo, a negociação envolve a determinação de quais riscos a "comunidade" aceita ou não correr quando a questão que se coloca é a circulação do "doente mental”. Um dos mecanismos dessa suposta negociação, que em muitos casos configura-se como coerção, é a estigmatização (BARBOSA, 2014, p.197).

Diante desse cenário, faz-se necessária a mobilização, em especial dos profissionais e estudantes de saúde, familiares de pessoas em sofrimento psíquico, para a redução da estigmatização das pessoas portadoras de transtornos mentais (VICENTE, 2013).

O modelo da atenção psicossocial exige rever o objeto de trabalho (constituir o sujeito enquanto cidadão), a prática (utilizar-se de novos instrumentos e com isso ampliar sua possibilidade de intervenção) e ampliar a finalidade da assistência, para além da remissão dos sintomas manifestos (AMORIM; CRUZ; CARDOSO, 2013).

É preciso investir em intervenções educacionais e na disponibilização de recursos em saúde mental de caráter generalizado e contínuo, de forma a se tornarem integrantes do cotidiano, da cultura das pessoas e das práticas em saúde pública, uma vez que intervenções pontuais, isoladas ou de curta duração têm demonstrado efeito limitado no combate ao hábito social e historicamente enraizado de estigmatizar os portadores de transtornos mentais (GUARNIEIRO et. al, 2012).

Em estudo realizado por Kirschbaum (2009), com o objetivo de identificar as características do processo de trabalho da enfermagem em um CAPS, a autora evidenciou a coexistência de conceitos diferentes, e até contraditórios, sobre o sofrimento psíquico e sobre o sujeito, que dão base à prática clínica, aos recursos terapêuticos e modelos tecnológicos. A autora ressaltou que a compreensão da equipe de enfermagem sobre seu papel no serviço de saúde mental conservava elementos da concepção introduzida, no século XVIII, pelo tratamento moral fundado por Pinel. 
Tecnologias leves para o cuidado de enfermagem na atenção psicossocial: contribuições à superação de estigmas sobre a doença mental

O objetivo de oferecer um atendimento singularizado, baseado na clínica ampliada e na reabilitação psicossocial, comprometidos com o desenvolvimento da autonomia e da qualidade de vida dos usuários, da família e da comunidade, deveria implicar a revisão dos instrumentos de trabalho da enfermagem. Se no modelo asilar esses instrumentos fixavam-se na vigilância, no disciplinamento e na tutela, deveriam ser substituídos, no novo contexto, pela escuta, acompanhamento terapêutico, grupos operativos e oficinas, e ainda, pelas atividades de referência, pautadas na singularização da atenção, mediante a elaboração de um projeto terapêutico individual (KIRSCHBAUM, 2009).

Ante ao exposto, faz-se mister estimular que os profissionais de saúde construam, numa perspectiva de cogestão com usuários e familiares, projetos terapêuticos singulares que considerem a sua experiência de existência-sofrimento, na compreensão dessa pessoa como sujeito ético, histórico e subjetivo; na aceitação do que diz e vive, de seus limites e possibilidades, permitindo-lhes novos projetos de felicidade (AYRES, 2006).

Diante da experiência vivenciada durante a execução do projeto de extensão "Interfaces Educação, Saúde e Cidadania: caminhos para a inclusão social dos usuários de um centro de atenção psicossocial" verificamos a necessidade de intervenções intersetoriais no município de Pesqueira, PE, envolvendo os profissionais de saúde de todos os níveis de atenção, os usuários do CAPS, seus familiares e toda a sociedade, a fim de favorecer a conscientização sobre as implicações do processo-saúde-adoecimento mental e sobre as concepções sobre a doença mental presentes na sociedade.

Ante ao exposto, o objetivo do presente artigo é relatar a experiência dos docentes e estudantes extensionistas no desenvolvimento de intervenções com vistas à superação de concepções estigmatizantes sobre o processo saúde-adoecimento mental, vivenciando a atuação do enfermeiro no âmbito da atenção psicossocial.

\section{MATERIAIS E MÉTODOS}

Trata-se de um relato de experiência descritivo, exploratório de abordagem qualitativa, de intervenções extensionistas realizadas no município de Pesqueira, durante os meses de fevereiro a dezembro de 2015. O projeto de extensão, que foi aprovado e cadastrado pela Pró-Reitoria de Extensão do IFPE, e autorizado pela gestão municipal de saúde, surgiu a partir dos resultados do projeto de pesquisa intitulado "Redes de Cuidado em Saúde Mental: o processo de construção da rede de atenção psicossocial no município de Pesqueira, PE”, que evidenciou concepções 
Tecnologias leves para o cuidado de enfermagem na atenção psicossocial: contribuições à superação de estigmas sobre a doença mental

estigmatizantes sobre o processo saúde-adoecimento mental, relacionadas à persistência da noção de risco como perigo, atribuídas às pessoas em sofrimento psíquico (BARBOSA, 2014).

No município de Pesqueira, no nível de atenção primária, os usuários são atendidos, principalmente pelas equipes da Estratégia Saúde da Família (ESF), Unidades Básicas de Saúde (UBS) e Núcleo de Atenção à Saúde da Família (NASF) por quem são referenciados para o serviço especializado, representado nesse município pelo CAPS II. No nível de atenção secundária, a Rede de Atenção Psicossocial (RAPS) de Pesqueira é composta pelo CAPS II, Policlínica, onde existe o atendimento ambulatorial de saúde mental, e Hospital Dr. Lídio Paraíba que atende aos casos de urgência e emergência psiquiátricas.

A ESF, juntamente com o CAPS, são capazes de oferecer um acolhimento condizente com as necessidades das pessoas em sofrimento psíquico, por estarem mais próximas do contexto social das famílias. Assim a ESF amplia o potencial dos CAPS como agenciadores de novos modos de cuidado pautados na corresponsabilidade pelo usuário e formação de vínculos entre a comunidade, família, usuários e a equipe de saúde. Portanto, o cenário escolhido para desenvolvimento das ações extensionistas foram duas Unidades de Saúde da Família (USFs), localizadas em áreas da cidade onde reside um grande número de usuários do CAPS II.

Realizamos intervenções de educação em saúde direcionadas aos profissionais de saúde e à comunidade adscrita as duas USFs, que problematizaram as concepções estigmatizantes sobre o processo saúde-adoecimento mental e o uso exacerbado de medicamentos psicotrópicos.

A metodologia escolhida para intervir junto às equipes de saúde e usuários das ESF foi o grupo focal (I-TECH, 2008). Um grupo focal é uma discussão estruturada para obter informação relevante de um grupo de pessoas, sobre um tópico específico. O objetivo do grupo focal é recolher informação sobre os sentimentos, valores e ideias das pessoas, e não obter consenso, nem tomar decisões (I-TECH, 2008).

Utilizamos também a proposta de educação popular de Paulo Freire como referencial teórico-metodológico para suporte às ações educativas propostas. De acordo com Linhares (2008), para Freire, uma educação popular e verdadeiramente libertadora, se constrói a partir de uma educação problematizadora, alicerçada em perguntas provocadoras de novas respostas, no diálogo crítico, libertador, na tomada de consciência de sua condição existencial. Tal investigação Freire chamou de "universo temático", um conjunto de "temas geradores" sobre os níveis de percepção da realidade do oprimido e de sua visão de mundo sobre as relações homens-mundo e homens-homens para uma posterior discussão de criação e recriação (FREIRE, 2004 apud LINHARES, 2008). 
Tecnologias leves para o cuidado de enfermagem na atenção psicossocial: contribuições à superação de estigmas sobre a doença mental

Demos início ao projeto de extensão realizando reuniões com os atores parceiros com o objetivo de apresentar a proposta de trabalho e ouvir sugestões para a continuidade das atividades, que foram desenvolvidas segundo as seguintes etapas:

I-Reuniões com as equipes de saúde do CAPS, ESFs, Secretaria de Saúde e NASF para o esclarecimento do nosso projeto.

II-Em conjunto às equipes acima mencionadas decidimos que nossas intervenções seriam dirigidas aos usuários que fazem uso contínuo de psicotrópicos, tendo em consideração registros de elevado consumo de benzodiazepínicos nessas comunidades. Verificamos que esses medicamentos também estão entre aqueles mais prescritos por médicos que não atuam no campo da psiquiatria, além das prescrições realizadas pelos próprios psiquiatras. Tendo em vista esse panorama, faz-se necessário problematizar o processo de prescrição e consumo dos benzodiazepínicos, bem como do consumo inadequado desses medicamentos por parte dos usuários, o que poderia propiciar um ambiente favorável ao uso continuado desses medicamentos (MANGINI JR, 2014).

III- Realizamos reunião de planejamento com nosso grupo de extensão e construímos a metodologia baseada na obra de Paulo Freire e na técnica de grupo focal.

IV- Como estratégia de estímulo à desmedicalização, optamos pelas Terapias Complementares e/ou Integrativas, denominadas pela Organização Mundial da Saúde como Medicina Tradicional. Compreendem um grupo de práticas de atenção à saúde não alopáticas e englobam atividades como a acupuntura, naturopatia, fitoterapia, meditação, reiki, terapia floral, entre outras. Estas terapias procuram atender ao indivíduo de forma holística, baseado na confiança e no vínculo terapeuta/usuário (GALLI et al, 2012).

Os agendamentos do grupo focal eram realizados pela equipe da ESF junto aos usuários, aproveitando-se o dia de cada mês em que os mesmos se dirigiam à USF para renovação da prescrição e/ou dispensação das medicações psicotrópicas. O grupo focal foi desenvolvido em sala ampla e de ambiente reservado, arejada, com iluminação natural, cadeiras dispostas em círculo. A sala era aromatizada com um vaporizador de ar com essências herbais. O grupo focal foi conduzido conforme os seguintes passos: 1) Convite e acolhimento aos participantes. 2) Técnica de respiração, relaxamento e meditação com som ambiente. 3) Realização das seguintes dinâmicas: 3.1) Cada usuário desenhava ou escrevia o que se sentia à vontade para expressar em um papel a respeito do que o medicamento representa em sua vida. Os desenhos foram então apresentados aos demais participantes, por cada usuário, e nesse momento eram socializadas as vivências, concepções e interpretações acerca dos remédios controlados; 3.2) Dávamos início a história de um personagem chamado Zé Orocó, extraída do romance "Rosinha, minha canoa" de 
Tecnologias leves para o cuidado de enfermagem na atenção psicossocial: contribuições à superação de estigmas sobre a doença mental

José Mauro de Vasconcelos (Edições Melhoramentos) e os participantes do grupo tinham que prosseguir com a história imaginando o que iria ocorrer com ele, construindo uma narrativa coletiva relacionada ao processo de tratamento dos transtornos mentais no modelo hospitalocêntrico e a transição para um modelo de saúde mental comunitário. Estiveram presentes muitas reflexões sobre o estigma social atribuído à pessoa portadora de transtornos mentais, ainda muito presente na comunidade de Pesqueira. 4) Utilizando-se como recurso visual um cartaz explicávamos sobre o uso de medicações psicotrópicas abordando sobre os seguintes subtemas: Riscos à Saúde, Riscos de Acidentes, Dependência, Recursos não medicamentosos para relaxamento substitutivos aos remédios controlados. 5-Avaliação: Os participantes avaliavam a atividade desenvolvida expressando uma palavra que resumisse sua participação no grupo focal e registrávamos suas impressões em um cartaz.

Cada grupo durava em torno de uma hora e foi conduzido por dois estudantes extensionistas do curso de enfermagem e, quando necessário, a professora orientadora e a enfermeira da ESF contribuíram para a condução dos grupos. Procuramos adotar uma linguagem compreensível para que a comunicação fosse efetiva com os participantes das intervenções.

A participação nos grupos se deu de forma espontânea, sendo garantido aos usuários e profissionais o sigilo quanto a sua identidade e opiniões, como também, retirar-se do grupo a qualquer momento, sem prejuízo à continuidade de seu atendimento na USF.

\section{RESULTADOS E ANÁLISES}

Com o aumento do número de CAPS, serviços prioritários substitutivos ao hospital psiquiátrico no processo de reforma psiquiátrica brasileiro, e a consolidação da política de saúde mental, voltada para a integração entre as ações de saúde mental com ênfase na atenção primária e secundária, o atendimento grupal é considerado um dos principais recursos terapêuticos nesses contextos (LANCETTI, 1993 apud SOUZA et. al, 2004). Portanto, os profissionais de saúde, dentre os quais o enfermeiro, precisam dedicar-se no sentido de estudar e implementar essa tecnologia leve para o cuidado em saúde mental.

No Brasil, a terapia de grupo em diferentes abordagens é praticada por grande número de profissionais de áreas diversas. O trabalho com grupos se constitui em um dos principais recursos terapêuticos nos mais diferentes contextos de assistência à saúde e, mais especificamente, no campo da saúde mental. Esse incremento decorre, em grande parte, das condições criadas a partir da reforma psiquiátrica, tendo por foco a ressocialização do indivíduo em sofrimento psíquico e a 
Tecnologias leves para o cuidado de enfermagem na atenção psicossocial: contribuições à superação de estigmas sobre a doença mental

horizontalização de saberes no processo terapêutico conduzido por uma equipe multiprofissional (SOUZA et. al., 2004)

Vale salientar que o uso de técnicas de relaxamento envolve cuidados adicionais relativos a estar atento ao ritmo do grupo e à possibilidade do relaxamento proposto induzir à evocação de vivências boas ou ruins e ao modo como trabalhar, na sequência, a assimilação e os significados das vivências. É importante considerar que, além das emoções despertadas pelo relaxamento, também ocorrem emoções geradas pela própria condução da técnica e condições do ambiente. Assim, privacidade, climatização do ambiente, tom de voz do coordenador e ritmo das músicas, todos são elementos que podem determinar o maior ou menor benefício do relaxamento (SOUZA et. al, 2004).

Durante nossas atividades optamos por utilizar música ambiente em tons calmos que favoreceram o exercício de concentração, bem como ajudaram no processo de realização da meditação que fizemos com os participantes.

Os dois estudantes extensionistas estiveram atentos às falas dos participantes, por essa razão não houve divisão entre guiar o grupo e observar ações e reações dos membros do grupo. Cada atitude pode demonstrar vários tipos de sentimentos ou sensações para os facilitadores. Conforme Souza et. al. (2004) os facilitadores devem estar atentos ao processo individual de cada participante e as várias emoções que surgem, podendo variar entre conforto e desconforto durante as atividades de relaxamento, ou durante as demais atividades de grupo. As expressões nos ajudam a avaliar como está sendo desenvolvido o grupo terapêutico e se as intervenções estão surtindo o efeito esperado (SOUZA et. al, 2004).

Outro resultado subjacente a nossas intervenções extensionistas foi a capacitação das equipes de saúde da família que foram coparticipes desde o planejamento até a avaliação das ações, experienciando um novo modo de cuidar das pessoas em sofrimento psíquico, não centrado no tratamento medicamentoso.

O desenvolvimento do grupo focal nas USFs permitiu-nos problematizar a questão do estigma atribuído à pessoa portadora de doença mental ou em sofrimento psíquico, assim como vivenciar a atuação do enfermeiro como mediador de grupos terapêuticos com ênfase em estratégias desmedicalizantes, como as práticas integrativas e complementares de saúde.

A Política Nacional de Práticas Integrativas e Complementares, lançada pelo Ministério da Saúde em 2016, tem o objetivo de proporcionar conhecimento sobre essas práticas e apoiar a implementação de experiências na rede pública de muitos municípios e Estados, entre as quais destacam-se aquelas no âmbito da Medicina Tradicional Chinesa-Acupuntura, da Homeopatia, da Fitoterapia, da Medicina Antroposófica e do Termalismo-Crenoterapia (MS, 2006). 
Tecnologias leves para o cuidado de enfermagem na atenção psicossocial: contribuições à superação de estigmas sobre a doença mental

O referencial teórico-metodológico freireano proporcionou que as atividades fossem desenvolvidas mediante o princípio da participação coletiva e dialogicidade, estabelecendo-se um rico diálogo entre o conhecimento científico e os saberes do senso comum, na construção de uma nova rede de sentidos sobre o processo saúde-adoecimento mental. Foi possível também a abordagem crítica sobre os avanços e limites do modelo de atenção psicossocial face à persistência de concepções vinculadas ao modelo asilar hospitalocêntrico.

No entanto, um aspecto não alcançado por nossas intervenções extensionistas foi a prescrição medicamentosa pela equipe médica da ESFs. Para Conrad (2007), o processo de medicalização através do qual problemas não médicos são definidos e tratados como problemas médicos, traduzindo-se em doenças, está vinculado a uma série de forças sociais e econômicas que sobre determinam a prática médica, ao que denomina de "motores da medicalização". Ao mesmo tempo, Conrad chama a atenção para o risco de se interpretar o termo "medicalização" como um movimento da corporação médica, afastando-se de uma compreensão mais ampla que considere sua dimensão processual. Nesse sentido, é preciso investir no processo de formação inicial e continuada dos médicos, assim como demais profissionais de saúde, para a superação de práticas de medicalização dos sofrimentos cotidianos.

Nessa direção, podemos considerar que uma repercussão positiva de nosso trabalho foi o estímulo às discussões nesse ambiente de saúde sobre o preconceito, estigma e dificuldades que os portadores de transtornos mentais enfrentam na sua rotina diária, o que na maioria das vezes é "invisível" aos olhos da comunidade e de profissionais, assim como sobre o processo de medicalização da sociedade.

Nossas experiências corroboram a afirmação de que precisamos investir num cuidado que reabilite as tecnologias leves, estimulando tudo o que há de potencial de vida e de autonomia. Por outro lado, é necessário, então, denunciar o crescimento exacerbado de tecnologias duras, representadas pelos insumos da indústria farmacêutica. "Cuidado que não exclua, violente ou discrimine os sujeitos, mas que construa possibilidades para a valorização de seus desejos e projetos", que contribua para a construção de novos sentidos para a loucura e para a crise, que não seja o do estigma e da exclusão, "mas firmados numa postura ética e solidária" (WILLRICH et al, 2011, p. 56). 
Tecnologias leves para o cuidado de enfermagem na atenção psicossocial: contribuições à superação de estigmas sobre a doença mental

\section{CONSIDERAÇÕES FINAIS}

Diante da centralidade que o tratamento medicamentoso adquire no processo de atenção à saúde mental ainda na atualidade, e da persistência, em alguns contextos, de práticas de cuidado vinculadas ao modelo asilar hospitalocêntrico, faz-se necessário investir em novas formas de cuidado em saúde mental, na direção de uma clínica ampliada, que contribua para uma nova compreensão do processo saúde-sofrimento-adoecimento psíquico, bem como para a invenção de práticas de cuidado, consoantes às proposições da Política Nacional de Saúde Mental e de acordo com as singularidades de cada território na RAPS.

Através das intervenções de extensão desenvolvidas foi possível refletir com os profissionais de saúde da família e membros da comunidade participantes dos grupos sobre seu papel na superação das concepções estigmatizantes sobre o processo saúde-adoecimento mental, que obstaculariza o processo de reinserção social dos usuários do CAPS II.

Evidenciou-se a relevância da atuação dos profissionais de enfermagem para a superação do ciclo social de estigmatização, como também, no desenvolvimento de estratégias de cuidado consoantes ao modelo da atenção psicossocial, tais como o acolhimento, os grupos terapêuticos e as práticas integrativas e complementares em saúde, tecnologias leves de baixo custo e, portanto, com grande potencial de inserção na APS.

Quanto aos estudantes extensionistas, vivenciaram a atuação do enfermeiro no âmbito da atenção psicossocial com ênfase no desenvolvimento de tecnologias leves para o cuidado em saúde mental na APS. Atuaram ativamente em todas as etapas, desde o planejamento coletivo até a implementação e avaliação, resultando na construção de habilidades e competências para o gerenciamento do cuidado no contexto da clínica ampliada.

Um aspecto limitante das intervenções extensionistas trata-se da dificuldade de prospecção dos resultados em termos de redução do consumo de benziodiazepínicos pelos usuários da USF, devido ao curto período em que as intervenções se deram, assim como da não participação do profissional médico da USF nas intervenções, uma vez que a adesão se deu espontaneamente, respeitando-se o direito dos sujeitos não se disponibilizarem a integrar os grupos terapêuticos. 
Tecnologias leves para o cuidado de enfermagem na atenção psicossocial: contribuições à superação de estigmas sobre a doença mental

\section{REFERÊNCIAS}

AMORIM, Ana; CRUZ, Diana; Cardoso, Maria. Percepção do Enfermeiro no Cuidar ao Doente Mental: Uma Revisão de Literatura. Rev Multip Saúde HSM. Teresina, v. 1, n. 2, p. 53-62, 2013.

AYRES, José Ricardo de Carvalho Mesquita. O Cuidado, os modos de ser (do) humano e as práticas de saúde. Saúde Soc, São Paulo, v. 13, n. 3, p. 16-29, set-dez, 2006. Disponível em: <http://www.scielo.br/. Acesso em 28 maio 2013

BARBOSA, Valquiria F. Bezerra. O Cuidado em Saúde Mental e o Dispositivo de Segurança no Brasil: transversalidades discursivas à problemática do Risco. Tese (Programa de Pós-Graduação Interdisciplinar em Ciências Humanas). Universidade Federal de Santa Catarina. Florianópolis, 2014. 191 p.

BRASIL. Ministério da Saúde. Política Nacional de Práticas Integrativas e Complementares no SUS - PNPIC-SUS: atitude de ampliação de acesso. Brasília, DF 2006. Disponível em: http://dab.saude.gov.br/portaldab/biblioteca.php?conteudo=publicacoes/pnpic Acessado em 25/06/2015.

CONRAD, Peter. The Medicalization of Society: on the transformation of human conditions into treatable disorders. Baltimore: The Johns Hopkins University Press, 2007. Disponível em: <http://books.google.com.br/>Acessado em: 01 jul 2015.

GALLI, Kiciosan et al. Saúde e equlíbrio através das terapias integrativas: relato de experiência.

Revista de Enfermagem FW. Santa Catarina, v. 8, n. 8, 2012. Disponível em:

http://revistas.fw.uri.br/index.php/revistadeenfermagem/article/view/491 Acessado em 25/06/2015.

GUARNIERO, Francisco et al,. O estigma da esquizofrenia na mídia: um levantamento de notícias publicadas em veículos brasileiros de grande circulação. Rev. Psiquiatr. Clínica. São Paulo, v. 39, n. 3, p. 80-84, 2012. Disponível em

$\leq$ http://www.scielo.br/scielo.php?script=sci arttext\&pid=S010160832012000300002\&lng=pt\& nrm=iso $>$. Acessado em 14 jun. 2015.

I-TECH. Department of Health and Human Services Health Resources and Services Agency (HRSA).Guião de implementação técnica 1. Organizar e Conduzir Grupos Focais. 2008. Disponível em: http://www.go2itech.org/resources/technical-implementationguides/1.TIG_Grupos_Focais_A4.pdf Acessado em 12/06/2015.

KIRSCHBAUM, Debora Isane Ratner. Nursing Agents' Perceptions on Their Work in Mental Health With Psychotic Patients in a Psychosocial Community Center. Rev. Latino-am Enferm, Ribeirão Preto, v. 17, n. 3, p. 368-373, mai-jun, 2009. Disponível em:<http://www.scielo.br/. Acesso em 12 maio 2012.

LINHARES, Luciano Lempek. Paulo Freire: por uma educação libertadora e humanista. PUCPR. 2008. Disponível em:

http://www.pucpr.br/eventos/educere/educere2008/anais/pdf/729 522.pdf

Acessado em 25/06/2015. 
Tecnologias leves para o cuidado de enfermagem na atenção psicossocial: contribuições à superação de estigmas sobre a doença mental

MANGINI JR, Zacarias Antonio; CAPONI, Sandra Noemi Cucurullo. Condicionantes relacionados ao uso crônico de clonazepam no Brasil: uma história de vida. Cad. de Pesq. Interdisc. em Ci-s. Hum-s. Florianópolis, Santa Catarina, v.15, n.106, p. 117-139, jan./jun., 2014.

MARTINS, Gisele da Conceição Soares et. al. O estigma da doença mental e as residências terapêuticas no município de Volta Redonda - RJ. Texto Contexto Enferm. Florianópolis, n. 22, v. 2, p. 327-34, abr-jun, 2013. Disponível em:

http://www.scielo.br/pdf/tce/v22n2/v22n2a08 Acessado em 10/06/2015

NUNES, Mônica; TORRENTÉ, Maurice de. Estigma e violências no trato com a loucura: narrativas de centros de atenção psicossocial, Bahia e Sergipe. Rev. Saúde Pública. São Paulo, n. 43 (Supl. 1), p. 101-108, 2009. Disponível em:

http://www.scielo.br/scielo.php?script=sci arttext\&pid=S0034-89102009000800015 Acessado em 10/06/2015.

SALLES, Mariana Moraes; BARROS, Sônia. Exclusão/Inclusão social de usuários de um Centro de Atenção Psicossocial na vida cotidiana. Texto Contexto Enferm. Florianópolis, n.22, v.3, p. 704-12, jul.-set., 2013. Disponível em: http://www.scielo.br/scielo.php?pid=S0104-

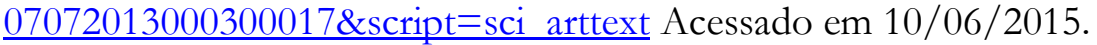

Representações sociais de usuários de um Centro de Atenção Psicossocial e pessoas de sua rede sobre doença mental e inclusão social. Saúde Soc. São Paulo, v.22, n.4, p.1059-1071, 2013. Disponível em: http://www.scielo.br/pdf/sausoc/v22n4/09.pdf Acessado em $11 / 06 / 2015$.

SOARES, Raisa Gontijo et.al. A mensuração do estigma internalizado: revisão sitemática da literatura. Psicologia em Estudo. Maringá, v. 16, n. 4, p. 635-645, out.-dez. 2011. Disponível em: http://www.scielo.br/scielo.php?pid=S1413-73722011000400014\&script $=$ sci arttext Acessado em 10/06/2015.

SOARES, Rosana de Lima. De palavras e imagens: estigmas sociais em discursos audiovisuais. Revista da associação nacional dos programas de pós-graduação em comunicação. Ecompós. Brasília, v.12, n.1, jan-abr, 2009.

SOUZA, Ângela Maria Alves et. al. Grupo terapêutico: sistematização da assistência de enfermagem em saúde mental. Texto Contexto Enferm. Florianópolis, v. 13, n.4, p.625-32, out-dez, 2004. Disponível em: http://www.scielo.br/pdf/tce/v13n4/a16.pdf Acessado em $20 / 10 / 2015$.

SPADINI, Luciene Simões; SOUZA, Maria Conceição Bernardo de Mello e. A doença mental sob o olhar de pacientes e familiares. Rev Esc Enferm USP. São Paulo, n. 40, v. 1, p.123-7, 2006. Disponível em: http://www.scielo.br/pdf/reeusp/v40n1/a17v40n1.pdf Acessado em $11 / 06 / 2015$.

VICENTE, Jéssica Batistela et.al. Aceitação da pessoa com transtorno mental na perspectiva dos familiares. Rev Gaúcha Enferm. v. 34, n. 2, p. 54-61, 2013. Disponível em:

http://www.scielo.br/pdf/rgenf/v34n2/v34n2a07.pdf Acessado em 10/06/2015. 
Tecnologias leves para o cuidado de enfermagem na atenção psicossocial: contribuições à superação de estigmas sobre a doença mental

WILLRICH, Janaína Quinzen et al. Periculosidade versus cidadania: os sentidos de atenção à crise nas práticas discursivas dos profissionais de um Centro de Atenção Psicossocial. Physis Revista de Saúde Coletiva, Rio de Janeiro, n. 21, v. 1, p.47-64, 2011. Disponível em:

$<$ http://www.scielo.br/. Acesso em 28 maio 2012. 